\title{
The Potential Roles of MELF-Pattern, Microvessel Density, and VEGF Expression in Survival of Patients with Endometrioid Endometrial Carcinoma: A Morphometrical and Immunohistochemical Analysis of 100 Cases
}

\begin{abstract}
Dmitry Aleksandrovich Zinovkin Md Zahidul Islam Pranjol ${ }^{1}$ Daniil Rudolfovich Petrenyov ${ }^{2}$ Eldar Arkadievich Nadyrov ${ }^{3}$ Oleg Gennadievich Savchenko ${ }^{4}$

University Research Laboratory, Gomel State Medical University, Gomel, Belarus; '1University of Exeter Medical School, Institute of Biomedical and Clinical Science, Exeter, Devon, United Kingdom; ¿Laboratory of Endocrinology and Biochemistry, Institute of Radiobiology National Academy of Sciences, Gomel; ' ${ }^{3}$ Laboratory of Clinical Research, Republican Research Center for Radiation Medicine and Human Ecology, Gomel; ${ }^{4}$ Department of Oncology, Gomel State Medical University, Gomel, Belarus
\end{abstract}

Received: May 14, 2017

Revised: July 6, 2017

Accepted: July 19, 2017

\section{Corresponding Author}

Dmitry Aleksandrovich Zinovkin, MD

University Research Laboratory, Gomel State

Medical University, Lange Str. 5, Gomel, Belarus

Tel: +375-29-182-7416

Fax: $+375-232-77-36-72$

E-mail: zinovkin2012@gmail.com

\begin{abstract}
Background: In this study, we hypothesized that microcystic, elongated, fragmented (MELF)-pattern, vascular endothelial growth factor (VEGF) expression by cancer cells and microvessel density of cancer stroma may be associated with progression of endometrioid adenocarcinoma. Methods: The study used data from the Belarus Cancer Registry and archival histological material of 100 patients with retrospectively known good (survival) and poor (disease progression and death) outcomes. All cases were immunohistochemically stained for CD34 and VEGF. Two independent samples were compared for the characteristics of signs, and obtained results were analyzed by receiver operating characteristic analysis, Mann-Whitney $U$ test, $\chi^{2}$ test (Yates correction), and Mantel-Cox test. Multivariate Cox hazard analysis and Spearman correlation test were used. A pvalue of less than .05 was considered statistically significant. Results: The observed survival rate of patients with endometrioid adenocarcinoma was significantly lower $(p=.002)$ in MELF-pattern positive patients when compared with MELF-pattern negative patients. The overall survival rate of patients whose tumors had more than 114 vessels $/ \mathrm{mm}^{2}$ of tissue was significantly low $(p<.001)$. Interestingly, a similar observation was found in patients with increased vessel area, evidenced by VEGF expression in the glandular tumor component. Conclusions: Our study suggests, for the first time, that these criteria may be used as risk factors of endometrioid adenocarcinoma progression during 5 years after radical surgical treatment. However, a large independent cohort of samples should be considered in the future to validate our findings.
\end{abstract}

Key Words: Carcinoma, endometrioid; Vascular endothelial growth factor; Prognosis; MELF; Vessel density
The stromal microenvironment of tumors is gradually becoming a main focus in the field of cancer research. It is believed that malignancy is a result of complex molecular and cellular interactions between the elements of tumor microenvironment and surrounding host tissues which induce selection and expansion of the neoplastic cells. ${ }^{1}$ In 2005 , Zigrino et al. ${ }^{2}$ reported an interaction of tumor cells with the stromal elements during tumor progression and paid special attention to the ability of the neoplastic cells to modify stroma by changing the adjacent connective tissue and modulating cellular metabolism of the host. In such circumstances, a new stroma is formed in areas of tumor invasion, including the parts of distant metastases, creating favorable conditions for the aggressive potential of tumor cells. This event in the areas of active cancer cell invasion is common in tumor progression. $^{2}$

Murray et al. ${ }^{3}$ introduced the acronym "MELF" (microcystic, elongated, fragmented) which describes the unusual changes incurred by the endometrioid adenocarcinoma (EA) when invading into the myometrium. These changes are characterized by the formation of microcysts lined with eosinophilic cytoplasm, elon- 
gated glandular structures, and clusters of individual cells. ${ }^{3}$ Though earlier it was believed that this fibromyxoid stromal reaction was initially a degenerative process, a number of studies have shown that the MELF pattern can be a specific tumor stroma reaction, similar to the epithelial-mesenchymal interactions observed in other tumors. ${ }^{4}$

Angiogenesis is defined by formation of new blood vessels from preexisting ones, playing a key role in uncontrolled proliferation of cells, survival of localized malignant cells and distant tumor invasion. Increased microvessel density, an indirect marker of intense tumor vascularization, is known to be associated with both evolution of the disease and patient survival. The formation of new vessels depends on the interaction between different hormones/growth factors and microvascular endothelial cells lining the existing microvessels. ${ }^{5,6}$ The endometrium expresses several growth factors involved in angiogenesis, including epidermal growth factor, transforming growth factor, and vascular endothelial growth factor (VEGF). VEGF is one of the most common promoters of angiogenesis, expressed even by the normal endometrium. As an angiogenetic factor, VEGF stimulates proliferation of endothelial cells and also increases vascular permeability and protein extravasations. $^{7-9}$

In this study, we hypothesized that MELF-pattern, VEGF expression by cancer cells, and microvessel density may be associated with the progression of EA and survival of patients. Therefore, we investigated the role of vessel and stroma of tumor microenvironment and reported for the first time that these criteria may be used as prognostic factors for $\mathrm{EA}$ of the uterine corpus.

\section{MATERIALS AND METHODS}

\section{Ethical approval}

The study was approved by the Committees for Medical and Health Sciences of Research Ethics of Republican Research Center for Radiation Medicine and Human Ecology and Gomel State Medical University. Dispensation from the requirement of patient consent was granted.

\section{Patient characteristics}

This retrospective study involved women with endometrial EA who were treated between January 2010 and December 2012 in the Grodno region, Republic of Belarus. The inclusion criteria for the study were stage I-III (International Federation of Gynecology and Obstetrics [FIGO], 2009), the presence of EA as the main cause of death, progression of the tumor, age between 45 and 80, hysterectomy, and an absence of malignant tumors in other parts of the body. The control subjects were selected cases of EA stage I-III with 5-year survival, age between 45 and 80, and no history of other malignant tumors. The exclusion criteria for the study were as follows: stage IV (FIGO, 2009), death from postoperative complications during the first month after hysterectomy, death from causes not related to EA progression, Lynch syndrome, synchronous and metachronous malignancies, and age less than 45 or more than 80 .

A total of 100 out of 156 cases of EA during the study period were determined to be eligible for inclusion in the study. The power analysis demonstrated that the number of patients was sufficient to conduct further research. The study used data from the Belarus Cancer Registry and archival histological material of 100 patients with histopathological diagnosis of EA. Histologic typing was performed according to the histological classification of endometrial cancer by the World Health Organization. Patients were divided into two groups. The first group included 48 subjects who had recurrence or died of EA within 5 years after diagnosis (unfavorable outcome). The second group consisted of 52 subjects who had no recurrence or death within 5 years after diagnosis (favorable outcome). The average age in the group with favorable outcome was $62.7 \pm 10.1$ years and it was $65.2 \pm 9.6$ years in the group with an unfavorable outcome. Patient characteristics by FIGO stage and tumor grade are presented in Table 1.

\section{Hematoxylin and eosin staining protocol}

Five-micrometer-thick sections were prepared from the archival histological blocks. They were mounted on microscopic slides. Next, the sections were deparaffinized in two portions of xylene and rehydrated in descending concentrations of ethanol. Subsequently, they were stained with hematoxylin and eosin by standard methods. The sections were cleansed in carbolic xylene, dehydrated in ascending alcohol concentrations, dried and mounted under coverslips using Biomount medium (DAKO, Glostrup,

Table 1. Characteristics of the patient groups by FIGO stage (2009) and tumor grade

\begin{tabular}{ccc}
\hline Characteristic & $\begin{array}{c}\text { Unfavorable outcome group } \\
(n=48)\end{array}$ & $\begin{array}{c}\text { Favorable outcome group } \\
(n=52)\end{array}$ \\
\hline FIGO & 20 & 23 \\
I & 23 & 23 \\
II & 5 & 6 \\
III & & \\
Grade & 17 & 16 \\
G1 & 25 & 31 \\
G2 & 6 & 5 \\
G3 & & \\
\hline
\end{tabular}

FIGO, International Federation of Gynecology and Obstetrics. 
Denmark).

\section{Primary antibodies and detection system}

Primary antibodies used in this study include the following: ready-to-use monoclonal rabbit anti-CD34 (clone EP88) and ready-to-use polyclonal rabbit anti-VEGF (Diagnostic Biosystems, Pleasanton, CA, USA). Mouse/Rabbit PolyVue Plus HRP/DAB Detection System (Diagnostic Biosystems) was used for primary antibodies visualization.

\section{Immunohistochemical staining protocol}

The 4-5- $\mu \mathrm{m}$-thick sections of tissue on l-polylysine coated glass slides were deparaffinized and washed with distilled water for 3 minutes. Antigen retrieval was performed using antigen unmasking solutions Tris-EDTA buffer ( $1 \mathrm{mM}, \mathrm{pH}$ 9.0) and citrate buffer $(1 \mathrm{mM}, \mathrm{pH} 6.0$ ), with preheating in the microwave at $800 \mathrm{~W}$ for 5 minutes and at $600 \mathrm{~W}$ for 10 minutes, respectively. The sections were then allowed to cool in the same solution. Endogenous peroxidase blocking was performed in 5\% hydrogen peroxide for 20 minutes, and blocking of nonspecific antibody binding was ensured by incubating the sections in 5\% casein in Tris-buffered solution for 1 hour. Following a brief wash in Tris-buffered solution, the sections were incubated in moist chamber at room temperature for 2 hours with corresponding primary antibodies. Tissue sections were then incubated at room temperature for 30 minutes with antimouse horseradish peroxidase secondary antibodies. Between each step the sections were washed twice with Tris-buffered solution for 5 minutes each. The reaction product was visualized with 3.3'-diaminobenzidine staining for 5 minutes, followed by Mayer's hematoxylin counter-staining. ${ }^{10}$

\section{Morphometry}

Determination of tumor vessels of microvasculature was carried out in the field with the largest number of capillaries (hot spots). The number and the area of vessels per $1 \mathrm{~mm}^{2}$ were determined by counting the number in 5 fields under the magnification of $\times 400$. These results were converted to $1 \mathrm{~mm}^{2}$ area of the tumor tissue. The microscope Nikon Eclipse 50i with digital camera DSF1 and NIS-Elements software (Nikon, Tokyo, Japan) was used for this morphometric work.

\section{Statistical analysis}

All data were presented by the median, lower and upper quartiles. A two-tailed Fisher test was used to compare the groups according to the presence or absence of MELF-pattern. MannWhitney test and receiver operating characteristic (ROC)-analysis were used for comparing the study groups based on the evaluated criteria. Determining the confidence interval (CI) and the area under the ROC-curve were the compulsory component of the ROC-analysis. The quality prediction model was labelled excellent at area under the curve 0.9-1.0, very good at 0.8-0.9, good at $0.7-0.8$, medium at $0.6-0.7$, and unsatisfactory at $0.5-0.6$. According to the threshold indicator, the patients were divided into two groups for 5 -year survival analysis by Mantel-Cox test. A Spearman correlation test was used for groups. A multivariate Cox proportional hazard analysis was developed using stepwise regression (forward selection, enter/remove limits $\mathrm{p}=.10$ ) to identify independent predictors of outcomes. A p-value of less than .05 was considered statistically significant. R v.3.4.0 free soft was used for statistical analysis.

\section{RESULTS}

\section{MELF-pattern}

Distinctive changes in the glands that characterized the MELFpattern were related with fibromyxoid stromal reaction. For instance, invasion of the myometrium by tumor glands showed that there is an absence of fibroblastic reaction (Fig. 1A). Intriguingly, fibromyxoid reaction compressing cancer glands were observed in the MELF pattern as expected (Fig. 1B).

The MELF-pattern was observed in eight cases $(16.7 \%)$ in the group with favorable outcome. Fibromyxoid changes were typical for the MELF-pattern which was observed in 17 cases (56.7\%) of EA in the group with unfavorable outcome. Statistical difference $(p=.014)$ was detected by comparing the number of the MELF-pattern present in the two groups. The observed survival rate of a patient with EA was significantly lower $(\mathrm{p}=.002)$ when MELF pattern was present compared with when MELF-pattern was not present (Fig. 2A).

\section{Number of vessels}

In the group with favorable outcome of the disease, the vessels were mostly detected in a small or moderate amount, with an ovalshaped lumen. Slight atypia was observed in the endothelium where the basement membrane was visualized throughout the cross-section of the vessels (Fig. 1C). On the contrary, the vessels of the microvasculature within the hot spot areas in cases with unfavorable outcome had mostly irregularly-shaped lumen. They were closely located to each other, often forming a densely branching network. It should be noticed that the endothelium had an irregular shape and an irregular intermittent basement membrane in the unfavorable outcome group (Fig. 1D). 

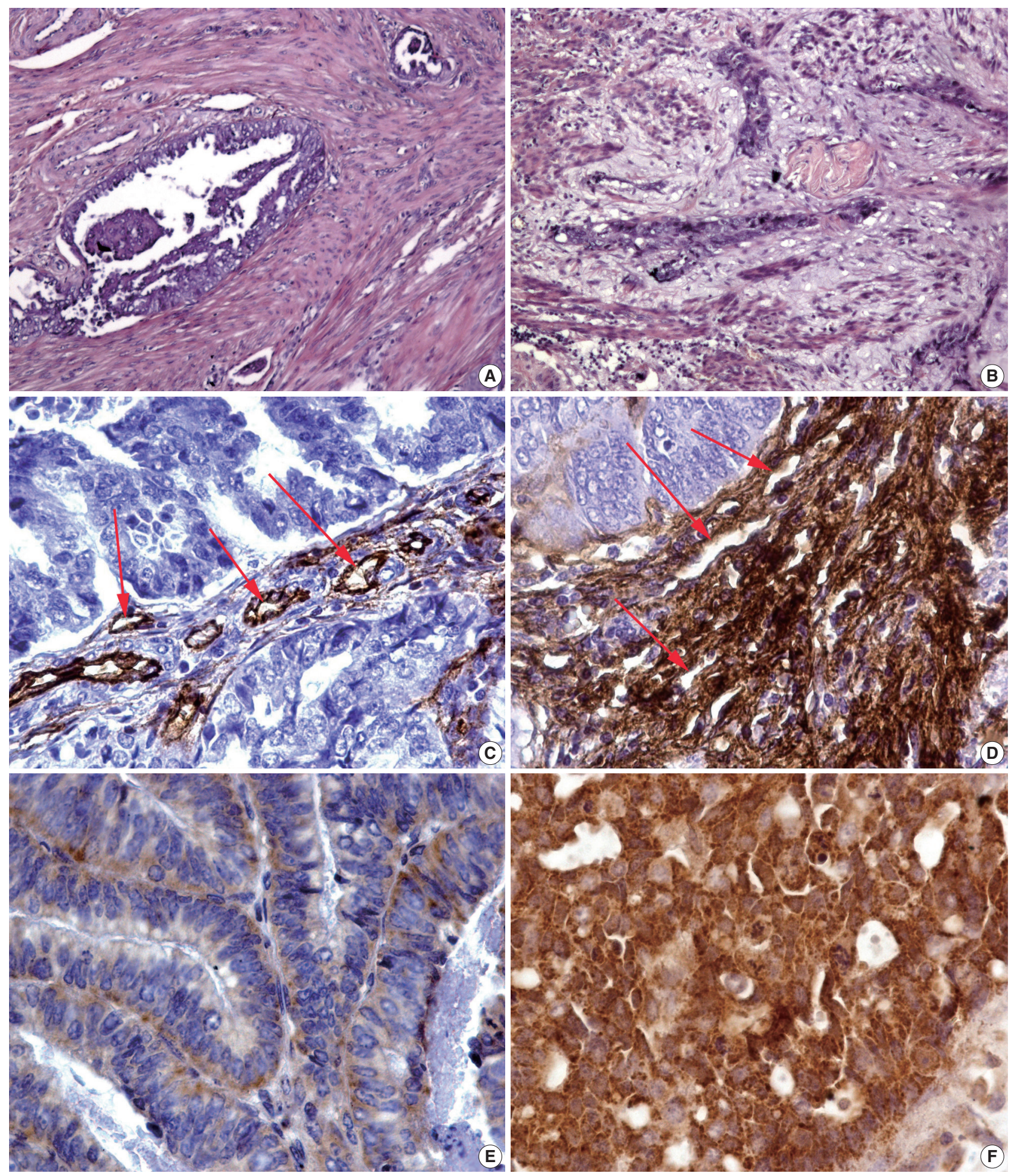

Fig. 1. (A) Stroma without fibroblastic reaction and tumor glands invading the myometrium. (B) MELF-pattern of the stroma with fibromyxoid reaction compressing the cancer glands. (C) Vessels in EA stroma with round lumen in group of patients with favorable outcome (arrows, CD34 immunostaining). (D) A large number of unusual vessels with dilated lumens in group of patients with unfavorable outcome (arrows, CD34 immunostaining). (E) Weak focal expression of VEGF in glands of EA, commonly detectable in patients with good outcome NEGF immunostaining). (F) Diffuse strong expression of VEGF in glands of EA in cases of unfavorable outcome (VEGF immunostaining). MELF, microcystic, elongated, fragmented; EA, endometrioid adenocarcinoma; VEGF, vascular endothelial growth factor. 

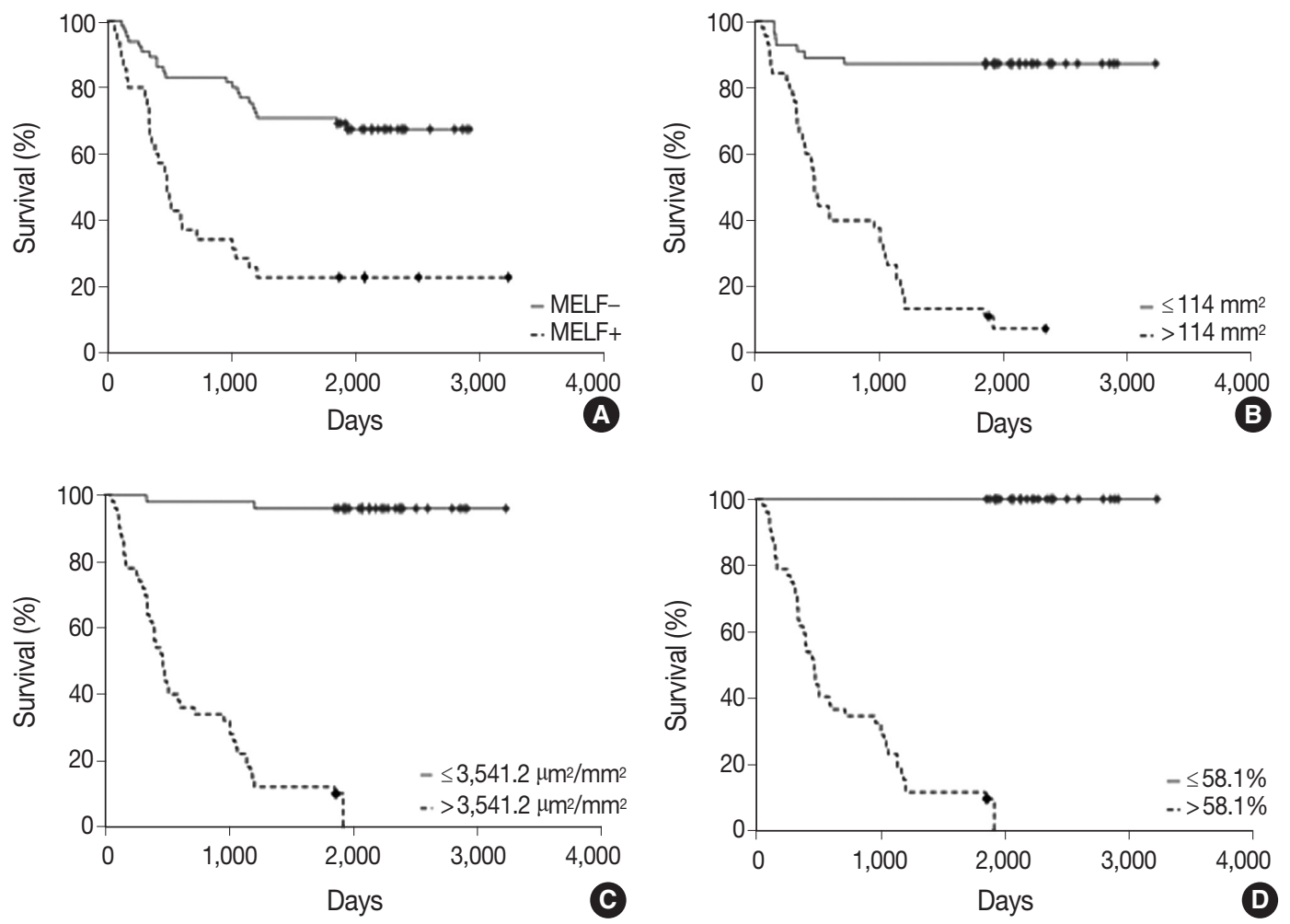

Fig. 2. Cumulative proportion survival. (A) MELF-pattern. (B) Number of vessels per $1 \mathrm{~mm}^{2}$. (C) Area of vessels per $1 \mathrm{~mm}{ }^{2}$. (D) VEGF expression by tumor glands. MELF, microcystic, elongated, fragmented; VEGF, vascular endothelial growth factor.

In the group of patients with unfavorable outcome, the median number of vessels in $1 \mathrm{~mm}^{2}$ of EA tissue was 139.1 (range, 74.1 to 174.6), and in the group with favorable outcome the median was 95.5 (range, 57.0 to 171.0). A significantly increased number of vessels were detected in the group with unfavorable outcome ( $p<.001 ; z=5.625$ ), compared to favorable outcome group.

The ROC-analysis of this index showed that the area under the ROC-curve was $92.3 \%$ (95\% CI, 82.5 to 97.6; $\mathrm{p}<.001$ ). The sensitivity was $86.7 \%$ (95\% CI, 69.3 to 96.2), the specificity was $96.7 \%$ (95\% CI, 82.8 to 99.9), and the threshold value of the index was 114.0 vessels $/ \mathrm{mm}^{2}$.

After studying the overall survival rate of patients with EA depending on the number of vessels in $1 \mathrm{~mm}^{2}$ of tumor, it was found that the survival rate was statistically lower $(\mathrm{p}<.001)$ in patients whose tumors had more than 114.0 vessels $/ \mathrm{mm}^{2}$ of tumor tissue (Fig. 2B).

\section{Area of the vessels}

The median of the vessel area in $1 \mathrm{~mm}^{2}$ of tumor tissue in group 1 was $4,904.1 \mathrm{\mu m}^{2} / \mathrm{mm}^{2}$ (range, 4,400.1 to $6,245.1 \mu \mathrm{m}^{2}$ ) $\mathrm{mm}^{2}$ ). The median of this index in the second group was 2,818.9 $\mu^{2} / \mathrm{mm}^{2}$ (range $1,348.2$ to $5,449.8 \mu \mathrm{m}^{2} / \mathrm{mm}^{2}$ ). A significantly larger area of vessels was detected in the unfavorable outcome group $(\mathrm{p}<.001, \mathrm{z}=6.247)$ compared with the favorable outcome group.

After performing ROC-analysis of the vessel area in $1 \mathrm{~mm}^{2}$ of tumor tissue, it was found that the area under the ROC-curve was $97.0 \%$ (95\% CI, 89.0 to 99.7; $\mathrm{p}<.001$ ). The sensitivity, specificity, and threshold value of the index were $100 \%(95 \%$ CI, 88.4 to 100.0 ), $96.7 \%$ (95\% CI, 82.8 to 99.9 ), and $3,541.2$ $\mu \mathrm{m}^{2} / \mathrm{mm}^{2}$, respectively.

The study of the overall survival rate of patients with EA depending on the vessel area of $1 \mathrm{~mm}^{2}$ of tumors showed that the survival rate was statistically lower $(\mathrm{p}<.001)$ in patients whose vascular area in tumors was more than $3,541.2 \mu \mathrm{m}^{2} / \mathrm{mm}^{2}$ (Fig. 2C).

\section{VEGF}

The expression of VEGF, one of the main stimulators of angiogenesis, was observed in all cases of EA. Diffuse expression of this marker was detected in the stroma and glandular component of EA. However, in cases of favorable outcome, a weak staining was observed in the cytoplasm, indicating a lower expression of VEGF (Fig. 1E). In the glandular component of the tumor, VEGF 
expression was, however, more evident and uniform. In the group with unfavorable outcome, an opposite result of immunohistochemistry was observed: the VEGF expression was strong, detected as brown staining foci in the cytoplasm of the tumor cells (Fig. 1F).

In cases with unfavorable outcome, the median of VEGF expression was $82.1 \%$ (range, $59.1 \%$ to $100.0 \%$ ) and it was statistically higher $(\mathrm{p}<.001 ; \mathrm{z}=6.616)$ in comparison with the median of cases with favorable outcome, which was $49.0 \%$ (range, $20.8 \%$ to $62.1 \%$ ).

The ROC-analysis of VEGF expression showed that the area under the ROC-curve was 99.8\% (95\% CI, 93.6 to 100.0; $\mathrm{p}<$ $.001)$. The sensitivity was $100.0 \%$ (95\% CI, 88.4 to 100.0$)$ and the specificity was $96.7 \%$ (95\% CI, 82.8 to 99.9). The threshold value of the index was $58.1 \%$. The survival rate was statistically lower $(\mathrm{p}<.001)$ in patients whose VEGF expression of the glandular tumor component was more than $58.1 \%$ (Fig. 2D), as expected.

\section{Correlation analysis}

Our study demonstrated a significant correlation between the MELF-pattern and VEGF expression in both groups. For instance, the correlation between the two was $r=.541(\mathrm{p}<.001)$. This was also observed between VEGF expression and the area of vessels $(\mathrm{r}=.762, \mathrm{p}<.001)$ and number of vessels $(\mathrm{r}=.648, \mathrm{p}<.001)$. Correlation analysis describes the changes in cancer stroma caused by VEGF expression in cancer cells.

\section{Multivariate Cox's proportional hazard model}

A multivariate Cox's regression analysis revealed that MELFpattern and the area and number of vessels per $1 \mathrm{~mm}^{2}$ of tumor tissue are independent prognostic factors of 5-year survival of patients with EA (Table 2).

\section{DISCUSSION}

Our study shows that the MELF pattern is more frequently observed in cases with unfavorable outcome than in those with favorable outcome, suggesting that the presence of MELF pattern may be a prognostic factor for patient survival. It can be assumed that MELF is a "medium" which enhances the spread of the tumor cells. After aggressive radiation therapy, a similar change of fibro-

Table 2. Multivariate Cox's proportional hazard model analysis of prognostic factors in patients with endometrioid adenocarcinoma

\begin{tabular}{lrcc}
\hline Factor & p-value & Hazard ratio & $95 \% \mathrm{Cl}$ \\
\hline MELF-pattern & .013 & 2.20 & $1.18-4.09$ \\
No. of vessels & .009 & 3.31 & $1.33-8.16$ \\
Area of vessels & $<.001$ & 1.03 & $1.01-1.17$ \\
\hline
\end{tabular}

$\mathrm{Cl}$, confidence interval; MELF, microcystic, elongated, fragmented. myxoid response in the stoma was observed in squamous cell carcinoma of the vulva, just as MELF pattern in EA. ${ }^{11}$ Immunohistochemical and genetic study of MELF pattern in EA disclosed stromal cell separation and disappearance and downregulation of E-cadherin expression. ${ }^{12}$ As pointed out by several authors, these changes are probably crucial in increasing the invasive capacity of EA and intensification of its metastatic potential. In the univariate analysis, the presence of stromal fibromyxoid reactions by MELF pattern was associated with an unfavorable prognosis of EA. ${ }^{8,10,11}$

The number and area of microvessels in our study showed statistically significant difference between the survival rates of patients with favorable outcome and unfavorable outcome. This can be used as a strong potential prognostic factor in the survival of patients with EA. Microvessel density in tumor-invaded tissue is increased by local angiogenesis that results in enhanced cancer cell proliferation during tumor progression. In gynecological cancer, angiogenesis is one of the crucial factors of tumor progression and plays a significant role in the maintenance of the growth of malignancies and their metastatic potential. ${ }^{13}$ Some authors suggested that the density of vessels of microvasculature is an indirect marker of the intensity of tumor vascularization, which is known to be associated with the progression of endometrial cancer and 5-year survival rate. ${ }^{14,15}$ In such reports, immunohistochemical marker CD34 was proven useful in the detection of these endothelial cells. ${ }^{16}$ The immunohistochemical expression of CD34 by endothelial cells allows counting the number and area of tumor vessels, which are prognostic signs that do not depend on other tumor characteristics, such as expressions of proliferation markers and adhesion molecules. ${ }^{17}$

Our study shows VEGF expression as a predictor of survival in patients with EA. For instance, the higher the expression of VEGF in cancer cells, the lower the survival of the patients, as predicted in our study. Nowadays, VEGF is the most frequently studied angiogenic promoter; its expression is observed in the normal endometrium as well as in other uterine malignancies, although it is higher in cancer tissue when compared with normal. ${ }^{18}$ VEGF stimulates endothelial cell proliferation, but it also increases vascular permeability, which helps the tumor cells to migrate to metastatic sites. ${ }^{19-21}$ Saito et al. ${ }^{22}$ reported that based on the surgical materials of 85 cases of EA, there was a significant VEGF expression in both highly differentiated and moderately differentiated tumors compared to poorly differentiated ones. In addition, it was reported that estrogen levels decrease the expression of VEGF, which may be an indication of increased survival of patients with EA. ${ }^{23}$ 
We showed for the first time that there is a significant presence of MELF pattern and an increased number and area of vessels in cases of EA with unfavorable outcome. In our study, VEGF expression correlated with the area and number of vessels, but it did not have any predictive force according to multivariate Cox's proportional hazard analysis. Although our data suggest that these criteria may be used as prognostic factors of EA during the 5 years after radical surgical treatment, a larger independent cohort of samples should be studied to verify these findings.

\section{Conflicts of Interest}

No potential conflict of interest relevant to this article was reported.

\section{REFERENCES}

1. Gacche RN, Meshram RJ. Targeting tumor micro-environment for design and development of novel anti-angiogenic agents arresting tumor growth. Prog Biophys Mol Biol 2013; 113: 333-54.

2. Zigrino P, Löffek S, Mauch C. Tumor-stroma interactions: their role in the control of tumor cell invasion. Biochimie 2005; 87: 321-8.

3. Murray SK, Young RH, Scully RE. Unusual epithelial and stromal changes in myoinvasive endometrioid adenocarcinoma: a study of their frequency, associated diagnostic problems, and prognostic significance. Int J Gynecol Pathol 2003; 22: 324-33.

4. Dogan Altunpulluk M, Kir G, Topal CS, Cetiner H, Gocmen A. The association of the microcystic, elongated and fragmented (MELF) invasion pattern in endometrial carcinomas with deep myometrial invasion, lymphovascular space invasion and lymph node metastasis. J Obstet Gynaecol 2015; 35: 397-402.

5. Kukreja I, Kapoor P, Deshmukh R, Kulkarni V. VEGF and CD 34: a correlation between tumor angiogenesis and microvessel density-an immunohistochemical study. J Oral Maxillofac Pathol 2013; 17: 367-73.

6. Żyła MM, Kostrzewa M, Litwińska E, Szpakowski A, Wilczyński JR, Stetkiewicz T. The role of angiogenic factors in endometrial cancer. Prz Menopauzalny 2014; 13: 122-6.

7. Stefansson IM, Salvesen HB, Immervoll H, Akslen LA. Prognostic impact of histological grade and vascular invasion compared with tumour cell proliferation in endometrial carcinoma of endometrioid type. Histopathology 2004; 44: 472-9.

8. Stefansson IM, Salvesen HB, Akslen LA. Vascular proliferation is important for clinical progress of endometrial cancer. Cancer Res 2006; 66: 3303-9.

9. Stewart CJ, Crook ML, Manso L. Fascin expression in low-grade uterine endometrioid adenocarcinoma: correlation with microcystic, elongated and fragmented (MELF)-type alteration at the deep inva- sive margin. Histopathology 2011; 59: 73-80.

10. Bajracharya D, Shrestha B, Kamath A, Menon A, Radhakrishnan R. Immunohistochemical correlation of matrix metalloproteinase-2 and tissue inhibitors of metalloproteinase- 2 in tobacco associated epithelial dysplasia. Dis Markers 2014; 2014: 197813.

11. Zaino RJ. Unusual patterns of endometrial carcinoma including MELF and its relation to epithelial mesenchymal transition. Int J Gynecol Pathol 2014; 33: 357-64.

12. Stewart CJ, Crook ML. Galectin-3 expression in uterine endometrioid adenocarcinoma: comparison of staining in conventional tumor glands and in areas of MELF pattern myometrial invasion. Int J Gynecol Pathol 2010; 29: 555-61.

13. Erdem O, Erdem M, Erdem A, Memis L, Akyol G. Expression of vascular endothelial growth factor and assessment of microvascular density with CD 34 and endoglin in proliferative endometrium, endometrial hyperplasia, and endometrial carcinoma. Int J Gynecol Cancer 2007; 17: 1327-32.

14. Aybatli A, Sayin C, Kaplan PB, Varol F, Altaner S, Süt N. The investigation of tumoral angiogenesis with HIF-1 alpha and microvessel density in women with endometrium cancer. J Turk Ger Gynecol Assoc 2012; 13: 37-44.

15. Haldorsen IS, Stefansson I, Grüner R, et al. Increased microvascular proliferation is negatively correlated to tumour blood flow and is associated with unfavourable outcome in endometrial carcinomas. Br J Cancer 2014; 110: 107-14.

16. Ozdemir O. Mast cell density, angiogenesis, and their significance in tumor development. Gynecol Oncol 2006; 100: 628-9.

17. Simionescu C, Mărgăritescu C, Stepan A, Pirici D, Ciurea R, Cernea $\mathrm{N}$. Tumor angiogenesis, macrophages and mast cell microdensities in endometrioid endometrial carcinoma. Oncol Lett 2013; 6: 415-20.

18. Nunobiki O, Nakamura M, Taniguchi E, et al. Adrenomedullin, $\mathrm{Bcl}-2$ and microvessel density in normal, hyperplastic and neoplastic endometrium. Pathol Int 2009; 59: 530-6.

19. Schmid BC, Oehler MK. Improvements in progression-free and overall survival due to the use of anti-angiogenic agents in gynecologic cancers. Curr Treat Options Oncol 2015; 16: 318.

20. Saarelainen SK, Staff S, Peltonen N, et al. Endoglin, VEGF, and its receptors in predicting metastases in endometrial carcinoma. Tumour Biol 2014; 35: 4651-7.

21. Wang J, Taylor A, Showeil R, et al. Expression profiling and significance of VEGF-A, VEGFR2, VEGFR3 and related proteins in endometrial carcinoma. Cytokine 2014; 68: 94-100.

22. Saito M, Sato Y, Watanabe J, Kuramoto H, Kaba S, Fukuda T. Angiogenic factors in normal endometrium and endometrial adenocarcinoma. Pathol Int 2007; 57: 140-7.

23. Matias-Guiu X, Davidson B. Prognostic biomarkers in endometrial and ovarian carcinoma. Virchows Arch 2014; 464: 315-31. 